\title{
Leishmaniasis in humans: drug or vaccine therapy?
}

This article was published in the following Dove Press journal:

Drug Design, Development and Therapy

\author{
Masoud Ghorbani \\ Ramin Farhoudi \\ Department of Viral Vaccine \\ Production, Pasteur Institute of Iran, \\ Research and Production Complex, \\ Karaj, Iran
}

\begin{abstract}
Leishmania is an obligate intracellular pathogen that invades phagocytic host cells. Approximately 30 different species of Phlebotomine sand flies can transmit this parasite either anthroponotically or zoonotically through their bites. Leishmaniasis affects poor people living around the Mediterranean Basin, East Africa, the Americas, and Southeast Asia. Affected regions are often remote and unstable, with limited resources for treating this disease. Leishmaniasis has been reported as one of the most dangerous neglected tropical diseases, second only to malaria in parasitic causes of death. People can carry some species of Leishmania for long periods without becoming ill, and symptoms depend on the form of the disease. There are many drugs and candidate vaccines available to treat leishmaniasis. For instance, antiparasitic drugs, such as amphotericin B (AmBisome), are a treatment of choice for leishmaniasis depending on the type of the disease. Despite the availability of different treatment approaches to treat leishmaniasis, therapeutic tools are not adequate to eradicate this infection. In the meantime, drug therapy has been limited because of adverse side effects and unsuccessful vaccine preparation. However, it can immediately make infections inactive. According to other studies, vaccination cannot eradicate leishmaniasis. There is no perfect vaccine or suitable drug to eradicate leishmaniasis completely. So far, no vaccine or drug has been provided to induce long-term protection and ensure effective immunity against leishmaniasis. Therefore, it is necessary that intensive research should be performed in drug and vaccine fields to achieve certain results.
\end{abstract}

Keywords: leishmania, leishmania treatment, vaccine, recombinant antigens

\section{Introduction}

Leishmania is an obligate intracellular pathogen that invades phagocytic host cells. ${ }^{1}$ Approximately, 30 different species of Phlebotomine sand flies can transmit this parasite either anthropologically or zoonotically by their bites. ${ }^{2}$

Leishmaniasis is endemic in 98 countries and about 350 million people are susceptible to the disease. ${ }^{3}$ Phenotypes of the disease include visceral, cutaneous, and mucocutaneous leishmaniasis (MCL). ${ }^{4}$ Cutaneous leishmaniasis (CL) is caused by Leishmania major and Leishmania tropica in the Middle East and central Asia and Leishmania braziliensis complex and Leishmania mexicana in Americas. Visceral leishmaniasis (VL, also known as the kala-azar disease) is caused by Leishmania infantum, Leishmania chagasi, and Leishmania donovani complexes. MCL is a severe and chronic mucocutaneous infection. ${ }^{5}$

Clinical manifestation of leishmaniasis can present with fever, anemia, wasting, hepatosplenomegaly, and suppression of immunity. Leishmaniasis predominantly affects poor people living around the Mediterranean Basin, East Africa, the Americas, and Southeast Asia. ${ }^{5}$

Different forms of leishmaniasis are more prone to occur based on the genetic background of the population. ${ }^{1}$ Several studies have shown that protectively immune responses against leishmaniasis are associated with induction of the T-helper 1
Department of Viral Vaccine Production, Pasteur Institute of Iran, Research and Production Complex, Kilometre 25 Autoban Karaj, 31599, Karaj, Iran Tel +982636102999

Fax +982636102900

Email ramin.farhoudi@yahoo.com
Drug Design, Development and Therapy 20I8:12 25-40

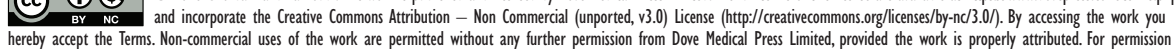

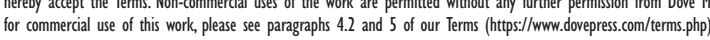


(Th1) cell cytokine interferon- $\gamma($ IFN- $\gamma){ }^{6}$ Along with this, neutrophils are essential cells to participate in inflammatory responses. ${ }^{7}$ Some studies have shown that Th1 cells with IFN- $\gamma$ and TNF- $\beta$ secretion are involved in immune responses against leishmaniasis infections. Other studies have shown that neutrophils can affect adaptive immune responses by chemokine production in leishmaniasis infections, thus resulting in the recruitment of other types of immune cells. Also, some studies indicate that any interaction between natural killer cells and dendritic cells with parasite reservoirs leads to host immune protection and destroys parasites through IFN- $\gamma$ production during $L$. major infections. ${ }^{7-13}$ Based on this and according to our understanding of these mechanisms of immunology, several vaccines have been designed but none of them had remarkable efficacy, and thus, have failed (Unpublished data).

Patients with VL die without any treatment. So, the most important aspect is the treatment in this field. Many drugs are used to cure these patients. But these drugs are limited because of high cost, toxicity, and other side effects. In addition, some studies have shown that drug resistance is a cause of relapse of infections in patients with leishmaniasis. ${ }^{14}$

Studies have shown that high cost, long treatment duration, and availability are major factors that increase the chance of drug resistance in underdeveloped countries. ${ }^{14,15}$ Considering these factors, drug therapy has not been effective to eradicate this disease. However, vaccination can provide long-term protection against disease and reduce transmission of infection. Therefore, extensive vaccination programs are required to reduce the incidence of leishmaniasis. ${ }^{16}$ However, the perfect vaccine is yet to be produced.

In the present review, we will discuss which therapeutic approach is most effective on leishmaniasis: drugs or vaccines? We will explore all related drugs and vaccines and conclude in accordance with our investigation.

\section{Drugs for leishmaniasis treatment}

Many drugs with various properties have their own benefits and limitations. First-line drugs include pentavalent antimony $\left(\mathrm{Sb}^{5+}\right)$, which has long been the basis of antileishmania chemotherapy. However, increasing resistance to the drug has restricted its benefits. ${ }^{17}$ Second-line drugs, such as pentamidine and amphotericin, are also used to treat this infection. However, emerging resistance and toxicity has stopped the use of pentamidine, while amphotericin B can induce acute toxicity and requires hospitalization. In addition, amphotericin B has another drawback in the form of high costs. ${ }^{18}$ Miltefosine, as an anticancer agent, was registered for the treatment of VL and CL. Oral efficacy and the short course of treatment were main advantages of this drug. However, it has major limitations in the treatment of leishmaniasis because of its teratogenicity effects and the long lifespan, which could favor promoting drug resistance. ${ }^{19}$ Chemotherapy is the most practical and effective treatment applied to all three major forms of leishmaniasis. But, as already mentioned, some unfavorable features of chemotherapy include toxicity, high cost, and long-term treatment, and thus new therapeutic ways must be devised to reduce these problems. ${ }^{5}$ Modes of action of these drugs include inhibition of mononuclear biosynthesis in amastigotes, disruption of parasite membrane, and intervention in the initiation of protein synthesis, among others. As a matter of fact, combination therapy of different drugs could be more effective and also has a shorter treatment duration and fewer side effects. Thus, a combination of amphotericin B with miltefosine has been recommended and prescribed for holding back antimonyresistant VL in India. However, there is a possibility that Leishmania could develop resistance to this combination as well. Adverse effects of current anti-leishmanial drugs are considerable. Cardiac arrhythmia, hepatitis, thrombophlebitis, and renal dysfunction were reported. In addition, improper use of medicine has led to drug resistance. ${ }^{20}$ We will discuss various kinds of these drugs in more detail.

\section{Pentavalent antimony $\left(\mathrm{Sb}^{5+}\right)$}

Sodium stibogluconate and meglumine antimoniate (MA), both pentavalent antimoniate compounds, have been used as key agents in VL and CL therapy under various conditions. The WHO recommends intralesional and systemic antimonials in the treatment of CL. ${ }^{21-23}$ Intralesional infiltration with pentavalent antimony creates a high concentration in the lesion, and there are a few side effects when injections are given; however, local administration as a negative point cannot affect metastatic injuries in far areas. ${ }^{24}$ According to the WHO-recommended therapy of local CL, 1-3 mL of pentavalent antimony solution should be injected under the lesion until the skin surface becomes pale. This administration method should be performed every 5-7 days for two to five times. As mentioned earlier, intralesional utilization of MA has been confirmed. ${ }^{25,26}$ In a case report, it was reported that a 45 -year-old man was bitten by sand flies and primary treatment was limited to suppressing acute allergic symptoms, and then the main treatment was performed by intralesional injection of 3-4 mL of the pentavalent antimoniate drug by which the lesions healed after 2 months. ${ }^{27}$ Therefore, intralesional injection is an optimum therapeutic approach. 
Benefits of this drug include low dosage, lower systemic side effects resulting in fewer complications, rapid action, the high concentration of drug glut lesions, and lower costs. Recently, some generic forms of pentavalent antimonite, such as sodium stibogluconate, are available to be used under different conditions. Also, MA has been used to treat VL intramuscularly and intravenously. ${ }^{27}$ According to reports, MA can affect enzymes in the liver and hence alanine aminotransferase (ALT) and aspartate aminotransferase (AST) levels were elevated temporarily in patients treated with MA. Also, CYP1A (Cytochrome P1A) activity has reduced with MA treatment in the mouse liver. Notably, $\mathrm{Sb}^{5+}$ and $\mathrm{Sb}^{3+}$ (potassium antimony tartrate) directly inhibit CYP1A catalytic activity in liver microsomes. However, any inhibitory effect is not observed at concentrations of up to $1 \mathrm{mM}$. Thus, $\mathrm{Sb}^{5+}$ treatment could not alter mouse CYP2A5 (Cytochrome P2A5), CYP2E1 (Cytochrome P2E1), and CYP3A11 (Cytochrome P3A11) levels. Nevertheless, constant administration of MA reduces CYP1A activity. In vitro studies have shown that both $\mathrm{Sb}^{5+}$ and $\mathrm{Sb}^{3+}$ do not alter CYP1A activity in mouse liver microsomes, while in vivo studies, where utilization of this drug can inhibit CYP1A catalytic activity, result in an enhanced risk of liver damage. Although MA compounds are being used in different forms of leishmaniasis, several reports indicate that resistance to the MA drug has increased. ${ }^{28}$ Earlier studies have shown that antimonials probably act with inhibiting glycolysis and fatty acid oxidation. ${ }^{29}$ The conversion of $\mathrm{Sb}^{5+}$ to $\mathrm{Sb}^{3+}$ can produce strong toxicity to kill the parasite, but resistance to antimonials has also been described with impaired reduction of $\mathrm{Sb}^{5+}$ to $\mathrm{Sb}^{3+}$. Also, this conversion will increase intracellular thiol and transmission of thiol-metal conjugates. Endemic studies have shown that Indian patients with VL could not respond to $\mathrm{Sb}^{5+}$ treatment efficiently. In this regard, folate and biopterin are reduced and catalyzed, respectively. And they are turned to biologically active forms by pteridine reductase enzyme. This could lead to intracellular life reduction and all the above-mentioned reasons of resistance to MAs. ${ }^{30-32}$ Furthermore, some studies have shown that cross-resistance can happen with interactions between $\mathrm{Sb}^{5+}$ and NO. As a result, inflammatory cytokines are produced and killing mechanisms in macrophages are inhibited. However, utility of this drug has been limited due to cardiac and renal toxicity in a number of patients with leishmaniasis. ${ }^{33}$ It could be concluded that the first-line drug for leishmaniasis has been used in developing countries for many years. But due to resistance problems in some nations, alternative therapeutic drugs without any resistance and more efficacy should be considered.

\section{Amphotericin B}

Amphotericin B deoxycholate (AmB-D) is considered as a second-line drug to treat leishmaniasis. Moreover, AmB drug in clinical treatment has been limited because of chronic and acute adversary effects such as fever, chill, nephrotoxicity, hypokalemia, myocarditis, and even death. This drug has very low bioavailability to treat orally, and thus it was utilized intravenously in systemic infections. Because of its insolubility in aqueous media, it is mixed with deoxycholate micelles (fungizone). However, the micelles are unstable in the bloodstream and AmB gets released; they, then, were transferred from micelles to lipoproteins into absorbing host cells, especially kidneys, so that free AmB is a contributing agent to toxicity. Hence, recent research has performed toward combining AmB composition into lipid-based drug-delivery systems to reduce its toxicity. As a matter of fact, this drug-delivery system decreases toxicity with the release of AmB slowly in monomer forms. Among all the proposed formulations, three forms have been found to have an emulsion, nanoparticles, and chemical modifications (and associations with other drugs). Obviously, each formulation of AmB is different in terms of morphology, composition, and biological activity. ${ }^{34-40}$

\section{AmBisome (AmB)}

Ambisome (liposomal amphotericin B) with a unique liposomal formulation can circulate in the body for a long period of time and penetrate tissues efficiently due to its small size. However, their loadings are very low. Also, AmB drug comprises unilamellar vesicles. Liposomes were widely evaluated for being used in drug delivery. They are spherical-shaped vesicles consist of an aqua core surrounded by lipid bilayers which possesses remarkable pharmacokinetic effects. Along with this, liposomes are designed to maximize antifungal activity and minimize the toxicity of the drug. Design of AmB within liposome together with an increased stability can enable the active compound to have a better engagement with pathogens in the various infection sites. The unilamellar L AmB lipid structure has three main components. At the first level, phosphatidylcholine is hydrogenated, which comprises most of the lipid bilayer. The second one, distearoyl phosphatidylglycerol, is adopted as its fatty acid branch, which is same in length to that of the hydrophobic area of amphotericin B and has a negative charge. AmB amino groups with positive charge form an ionic complex with distearoyl phosphatidylglycerol. Therefore, it could enhance $\mathrm{AmB}$ retention within the lipid bilayer. The third component, cholesterol, is added to AmB which facilitates the retention 
of this drug within the liposome bilayer. ${ }^{41-44}$ In this regard, the liposomal formulation of amphotericin B (LAmB) is limited to special conditions, such as children $<1$ year old and elderly individuals. In other reports, we found that the utility of a high dose of LAmB drug can be successful up to $90 \%$ in CL and VL treatments within a very short period of time and no remarkable complications were observed. Nevertheless, the price of the LAmB is high. Because of this, developing countries, such as Brazil, use first and second lines of treatment versus LAmB. ${ }^{45}$ Comparing these two drugs (AmB-D and LAmB) at a glance, some important parameters should be considered. These parameters are size, molecular weight, pharmacokinetic $\mathrm{Cl}$, renal clearance, terminal half-life second phase, distribution, clinical US Food and Drug Administration (FDA)-approved indications, and nephrotoxicity risk and cost. The size of AmB-D is $<0.04$, but LAmB is $0.08 \mu \mathrm{m}$. The molecular weights of AmB-D and LAmB are the same and equal to $924 \mathrm{~g} / \mathrm{mol}$. Pharmacokinetic effects of AmB-D in CL is $13.1 \pm 2 \mathrm{~mL} \mathrm{~kg} / \mathrm{h}$, but in LAmB, it is $9.7 \pm 5.4 \mathrm{~mL} \mathrm{~kg} / \mathrm{h}$. Renal clearance of AmB-D is $4.1 \pm 0.68 \mathrm{~mL} / \mathrm{h} / \mathrm{kg}$ but of LAmB is $0.495 \pm 0.25 \mathrm{~mL} / \mathrm{h} / \mathrm{kg}$. The terminal half-life second phase of AmB-D is 10-24 h but in LAm-B, it is 6-23 h. The distribution of AmB-D is liver $>$ spleen $>$ lung $>$ kidney, but in LAmB it is spleen $>$ liver $>$ kidney $>$ lung. Clinical FDA-approved indications of AmB-D are cryptococcosis, blastomycosis, candidiasis, coccidioidomycosis, histoplasmosis, mucormycosis, and aspergillosis, respectively, but in LAmB they are aspergillosis, candidiasis, cryptococcal meningitis, VL, and prolonged fertile neutropenia, respectively. Nephrotoxicity risk of AmB-D is high, but it is moderate in LAmB. The cost of AmB-D is low ( $£ 3.88$ per $50 \mathrm{mg}$ vial) and of LAmB it is high ( $£ 82.19$ per $50 \mathrm{mg}$ vial). ${ }^{40,46-50}$ One study performed on the mice model with VL and response therapy to LAmB was evaluated. The result of this experiment showed that a dose of $0.8 \mathrm{mg} / \mathrm{kg}$ reduced the parasite level in the liver and the spleen, while utilization of dosages of 5 and $50 \mathrm{mg} / \mathrm{kg}$ of this drug resulted in complete elimination of the liver, spleen, and lung. Several clinical studies indicated that the best result of using LAmB in VL infection could arise at the dose of $20 \mathrm{mg} / \mathrm{kg}$ as recommended by WHO. ${ }^{40}$

Notably, with this regard, we ought not to ignore the effects of AmB-D on people being treated with leishmaniasis. Several studies have shown that treatment with AmB-D is more effective than $\mathrm{Sb}^{5+}$ (usually injections of $\mathrm{Sb}^{5+}$ and AmB-D are performed intravenously) and patients treated with AmB-D should be monitored accurately with daily appraisals to facilitate health problems. It is noteworthy that AmB-D related toxicities occurred frequently but were potentially preventable with adequate monitoring, supplemental fluid and electrolyte therapies and the proportion of patients treated with AmB-D based therapy has increased from $30 \%$ to $80 \% .{ }^{51,52}$ Based on the above-mentioned factors in which safety and efficacy of LAmB are more excellent than first and second lines of treatment, it is questioned why LAmB is not considered as a first-line drug to treat leishmaniasis? To answer this question, some pharmacoeconomic studies should begin to evaluate the cost of taking this drug versus $\mathrm{Sb}^{5+}$ and AmB-D. The results of a few pharmacoeconomic analysis showed that the total cost of treatment by the LAmB equals to that of $\mathrm{Sb}^{5+}$ and AmB-D. Some evidence in the literature supported this result. However, clinical comparative studies have not yet been performed to convince us to support the change of treatment guideline. ${ }^{53-56}$ Another answer, which would be imagined about LAmB, is associated with low renal toxicity when compared with free $\mathrm{AmB}$ so that $\mathrm{AmB}$ is bounded to lipoprotein ligand with low density, which is present in the kidney abundantly. However, liposomal-encapsulated AmB has top affinity for liposomal receptors, which are expressed in the reticuloendothelial system (RES) at high concentration and result in reduced renal toxicity. The RES system could phagocytosis LAmB to modulate tissue distribution in such a way that the LAmB complex can access the tissues with an increased level of AmB. After degradation of the LAmB complex in the tissues, free AmB could return to plasma and gets redistributed in tissues again. High accumulation of liposomal-encapsulated drugs in tissues could not lead to toxicity in tissues because these drugs get segregated in safety tissue compartments, such as macrophages. ${ }^{57-60}$ Several observations convey the concept that uncontrolled distribution of AmB drug damages normal tissues, but, as mentioned earlier, the minimal toxicity in LAmB utilization was seen. Thus, we found that the liposomal system with suitable features can enhance these properties efficiently with a combination target-specific ligand on the surface of the system. Although accessible liposomal formulations of AmB target leishmaniasis and other parasite infections effectively, they show elevated exposure to all the tissues in body and their loadings are almost low. Therefore, the drug-delivery system should be optimized via receptor mediated so that the use of carbohydrate ligands to target protein receptors at the site of localization can play important roles in targeted drug delivery. Studies have shown that polysaccharide-modified ligands were combined in liposomes to make surface-modified liposomes (SML). The results of this evaluation indicated that SML, in 
combination with polysaccharide-modified ligands, enhance anti-leishmanial efficacy of AmB to target macrophages and RES successfully. ${ }^{61}$ As mentioned above, LAmB is expensive, but a rational approach has been noticed, which includes utilization of drug-targeted delivery systems via a variety of anchors. Hence, studies have reported that chitosan acts as an anchor to combine with doxorubicin in $\mathrm{AmB}$ and can be used as a macrophages-targeting drug-delivery pathway for VL. Chitosan-coated nanoparticles produce the "eat me" signal for specified phagocytes and result in drug internalization into the cells. The effect of this established approach and its benefits were illustrated with minimal toxicity, followed by slow delivery targeted to macrophages. ${ }^{62}$ Along with others, AmB formulations are used in clinical treatments including AmB lipid complex (ABLC) and AmB colloidal dispersion $(A B C D)$. These formulations are significantly different at the point of compositions and pharmacokinetic properties. ABLC is formed of ribbon-like multi-lamellar and flattened structures with particles 1,600-11,000 nm in length that cause a bigger volume distribution. Plasma concentrations of AmB have decreased, followed by ABLC, when compared with LAmB. ABCD is a complex of AmB and cholesteryl sulfate that is composed of thin disk-shaped structures. The size of this drug is $120 \mathrm{~nm}$ in diameter, and is immediately removed from circulation by RES. Along with this, the big size of the ABCD drug could be a limitation factor to treat disseminated diseases. ${ }^{40,63}$ However, LAmB has been the only approved drug by FDA. Variation in geography is one more reason to determine how the dose should be given. Based on this aspect, the total dose of $10 \mathrm{mg} / \mathrm{kg}$ has caused $>95 \%$ of cure rates in India. In Mediterranean and South American areas, a total dose of $18-21 \mathrm{mg} / \mathrm{kg}$ is recommended, but a higher dose of LAmB is required to treat VL in East Africa. Some studies in Sudan have shown that a dose of $30 \mathrm{mg} / \mathrm{kg}$ results in a cure rates of $>92 \%$ with $1 \%$ failure in treatment, $5 \%$ death, and $7 \%$ relapses. ${ }^{64}$

\section{Resistance to amphotericin B}

Resistance to amphotericin B in clinical isolates of L. donovani has been found. These clinical isolates are without any ergosterol, but they have replaced exogenous cholesterol combined into their membrane. AmB-resistant Leishmania strains showed significantly reduced reactive oxygen species (ROS) accumulations, which lead to increased tryparedoxin cascade. Along with this, the pathway could play a vital role in antioxidative defence against ROS in kinetoplastides. The result of the proteomic analysis showed that the AmB-R strain of L. infantum can upregulate members of the tryparedoxin cascade. This proteomic study revealed that reduction of an ATPase $\pm \mathrm{H}+$ in the AmB-R Leishmania strains has led to promoted proton permeability across $\mathrm{AmB}$ aqueous pores and because of the consequent waste of ATP has led to elevated ROS accumulation. Resistance to AmB is associated with ATP depletion, which was followed by an increase in ion leakage. By preventing ion leakage, we could inhibit ATP depletion and better control AmB-R Leishmania strains. Other studies also found that the ATP-binding cassette (ABC) family of transporters and various Ras isoforms probably have undeniable roles in AmB-R strains of Leishmania. ${ }^{65-68}$

All the above-mentioned factors show us that the secondline treatment with amphotericin B with suitable features could be effective partially to inhibit leishmaniasis growth. However, due to high toxicity and treatment resistance, it has limited clinical use in this respect. On the other hand, with modification of $\mathrm{AmB}$ to reduce its toxicity by changing the liposomal formulation and utilization of the drug-delivery system could be profited by their benefit in clinical utilization. But the price of L-AmB is very high, especially in developing countries. Finally, according to studies, the liposomal form of AmB drug can be adopted as a first-line drug to treat patients in the developed countries. Nevertheless, the use of this method is not affordable in the developing countries.

\section{Miltefosine as third-line treatment}

The chemical name of miltefosine is hexadecyl 2-ethyl phosphate, and it is considered as a third-line anti-leishmanial drug. Miltefosine as a zwitterion and amphiphilic compound with $407.57 \mathrm{~g} / \mathrm{mol}$ molecular weight is used to treat VL and CL diseases through the oral route. ${ }^{69,70}$

The most reported side effect is gastrointestinal discomfort, which is often a poor reason for treating these infections. Although miltefosine had an excellent activity on trypanosomatid parasite, its superiority was reached in the development of a local treatment for metastatic breast cancer and, consequently, this formulation was approved. Miltefosine application was evaluated in oral formulation for treating solid tumors and the results showed that consumption of the miltefosine drug has failed because of its side effect mentioned earlier. In vitro findings on the Leishmania parasite demonstrated that high bioavailability of miltefosine has a priority role versus parenteral $\mathrm{Sb}^{5+}$. In a history point of view, the first Phase II study of oral miltefosine for VL treatment in India had promising results. As a result, miltefosine drug was approved in 2002 as a first oral drug to treat leishmaniasis. The drug miltefosine inhibits the AKt/PKB signaling pathway, which involves cell survival. Recently recommended 
dosage of miltefosine to treat VL and CL is $2.5 \mathrm{mg} / \mathrm{kg} /$ day for 28 days. But this dosage varies in different countries for example, in India the recommended dose is $100 \mathrm{mg} /$ day for patients with body weight $<25 \mathrm{~kg}$ and it is $50 \mathrm{mg}$ /day for patients with body weight $>25 \mathrm{~kg}$. The cost of 56 capsules containing $50 \mathrm{mg}$ is between $£ 45.28$ and $£ 54$.92. In preclinical in vitro studies, no oxidative metabolisms were observed by isoenzyme CYP450 (Cytochrome P450). The main metabolic pathway of miltefosine is exerted via phospholipases. Furthermore, in vitro data showed that phospholipase $\mathrm{C}$ was able to hydrolyze miltefosine, and it has more than one site of action. ${ }^{70-73}$

\section{Resistance to miltefosine}

Parasites resistant to miltefosine could be created as promastigotes by chemical mutations. Imperfection in drug internalization due to mutations in MT gene is considered as the resistance mechanism. This fault relating to the accumulation of the drug could be revised with the functional miltefosine transporter gene expression to resist parasites. Besides, single mutations in Ros3 gene alleles were seen. These data suggest that miltefosine transporter activity is essential and inactivation of this transporter can lead to parasite resistance to miltefosine. Also, miltefosine resistance could be related to increased efflux pump via overexpression of $\mathrm{ABC}$ transporter. The results showed that miltefosine could be a suitable alternative for $\mathrm{Sb}^{5+}$ to treat L. major $^{74-77}$ Thus, it should be considered that the above-mentioned side effects of miltefosine have limited clinical use in the treatment of leishmaniasis. Alternatively, paromomycin, an aminoglycoside that can interfere with protein synthesis through $16 \mathrm{~S}$ RNA, could be used as an efficient anti-leishmanial drug. ${ }^{78}$ However, high cost and remarkable side effects of these single therapies forced us to shift to other therapeutic options. But before that, we should investigate combination therapy in this field.

\section{Combination therapy}

In a preclinical study, miltefosine activity was enhanced when it was combined with AmB drug. With this strategy, the treatment duration was reduced, which resulted in decreased therapy cost. Also, the treatment efficacy for complicated cases was improved and parasite resistance was delayed. Several studies have shown that a combination of miltefosine with a single injection of LAmB (5 mg/ $\mathrm{kg}$ ) could lead to reducing treatment lifespan with miltefosine solitary from 28 days to 7 days without any decrease in drug efficacy. In 2002, in India, miltefosine was the first oral therapeutic agent used to treat VL. But considering that miltefosine is not a cost-effective solution, usually, this drug in combination with paromomycin or LAmB was used, which could be a cost-effective therapy for patients. ${ }^{70}$

Although the mechanism of action of anti-leishmanial drugs could inhibit infection growth, patients suffer from serious adverse effects of these drugs. Combination therapy could offer promising results for $\sim 98 \%$ of the cases and reduced resistance issues significantly in some clinical trial studies and in endemic areas such as in India as well. However, this treatment strategy could not be generalized for all the therapeutic aspects in other countries. Thus, designing a perfect drug with $100 \%$ efficacy to eradicate any kind of leishmaniasis without remarkable toxicity remains unachieved. ${ }^{79}$

Based on the above mentioned paragraph, the treatment of leishmaniasis was usually abandoned due to the inevitable side effects of drugs as well as improper use of medicine which has led to drug resistance. So, drug therapy cannot eliminate leishmaniasis completely. Eventually, a perfect drug has not yet been designed, and it seems that R\&D (research and development) is responsible to perform more researches in this regard to produce new anti-leishmanial drugs with powerful effect, optimum performance, and also without any toxicity. ${ }^{20}$

\section{Vaccine against leishmaniasis}

Vaccination strategy is the most economical method for the prevention of infectious diseases. Generally, there are different forms of vaccines: killed, attenuated, recombinant, subunit, VLP (virus-like particle), and DNA vaccines. Preventive vaccines with induction immune responses can produce memory lymphocytes toward the immunity pathway for controlling infections. Also, these vaccines stimulate humoral and cellular immunity, especially a strong Th1 response and also cytotoxicity cells, to eliminate infections..$^{20,80}$

However, such vaccines for preventing leishmaniasis are yet to be found. Of course, some combination therapy has been presented for leishmaniasis HIV-positive patients.

Many studies have shown that sand fly has immunogenic proteins in saliva, which could induce immune responses as an adjuvant. In rodent models of infection, salivary molecules could induce immunity to protect against both CL and VL. Salivary molecules can negatively impact the parasites because of being adjacent to the site of infection bite. In other studies, immunized BALB/C mice with secretory serine protease plus IL-12 were utilized as an adjuvant to induce a strong Th1 response. They showed that this immunization could reduce the parasite load in spleen and 
liver and increase the IgG level in serum, which suppressed the promotion of the disease. As a result, use of an effective adjuvant to induce comprehensive immunity is considered in mouse model studies. ${ }^{81}$ In another study, Giunchetti et al selected two groups of dogs to evaluate the antigenicity of a whole vaccine. Their result showed that $\mathrm{CD}^{+} \mathrm{T}$-lymphocyte and $\mathrm{IgG}$ antibody increased. ${ }^{82}$ In fact, we found that vaccine preparation needs to adopt an optimum adjuvant for enhancing immunogenicity. Formulating vaccine with an adjuvant will determine what kind of immunity is generated. The best adjuvant stimulates both humoral and cellular immune responses.

In a clinical trial, Khalil et al evaluated autoclaved L. major (ALM) as a vaccine in a double-randomized trial. They showed that ALM does not have any significant protective immunity. However, adoption of suitable adjuvant would be helpful to induce the maximum protective immunity with the minimum side effect. ${ }^{83}$ In another study, Jay et al worked on recombinant Leishmania poly-protein LEISH-F1 antigen plus MzPL-SE as an adjuvant in the clinical trial. They showed that the vaccine was safe and can induce T-cell production of IFN- $\gamma$. Results of the clinical trial showed that this vaccine is immunogenic and safe in healthy individuals with and without previous history of infection. However, study population was small in this trial and trials with large population are required to appraise the efficacy of LEISH-F1 + MPL-SE. ${ }^{84}$

Although some clinical trial studies have reported promising results, it is necessary to design perfect vaccines with high immunogenicity and safety to induce immune responses, especially Th1 and cytotoxicity T cells, by suitable adjuvant adoption for intriguing antigen-presenting cells (APC) activity.

In this regard, use of whole parasites and raw antigen with suitable and optimum adjuvants has provided the adequate circumstances for vaccination to protect from leishmaniasis infection. ${ }^{85-88}$ There is considerable difficulty in standardizing and optimally formulating raw preparations to selectively induce appropriate immune responses. But, in the variable nature of the preparations of these vaccines, contradictory results may have been obtained in clinical trials. To solve this problem, proteins produced by recombinant methods were utilized in relation with appropriate adjuvants. According to the recombinant nature of this vaccine, it is accessible to large scale, reproducible and cost-effective production. Several antigens have been suggested and appraised as a vaccine candidate for different forms of leishmaniasis. Some studies have reported that varying status of protection in mouse model of $L$. donovani infection could be due to several antigens. ${ }^{89-92}$ Two criteria that should be considered to initially select potential vaccine antigens include a known antigen that expressed in the disease-causing mammalian stage of the parasite, and the selected adjuvants that elicit a cellular, Th1-biased immune response for the immunizations in humans. ${ }^{16}$ Side effects of an adjuvant, such as toxicity, should be noted for the formulation of the vaccine. Studies have shown that adjuvants adopted for vaccine formulation are considerably important because some adjuvants could shift immune responses to different pathways and other precautions. ${ }^{93}$

\section{First-generation vaccines Prophylaxis}

First-generation vaccines against leishmaniasis include vaccines made of whole killed parasites. These vaccines can be produced with low cost in developing countries. This is one of the benefits that attract some candidates for vaccine development. But there are many potential obstacles to the registration of standardization of vaccines derived from cultured parasites. Of late, three forms of vaccines consisting of L. major, Leishmania amazonensis, and L. mexicana were evaluated by first-generation vaccines of human clinical trials. ${ }^{94}$

\section{Efficacy trials of first-generation vaccines}

By early 20th century, first-generation vaccines were tested in a trial in Latin America. Two main vaccines were appraised in the world. One of them was pentavalent preparation by Armijos et al in Brazil. Parasite inactivation was performed using merthiolate and the vaccine, known as Leishvaccine, was without any adjuvant. The other was a preparation of autoclaved similar vaccine, which gave the same results of immunogenicity as well. L. mexicana mixed with Bacillus Calmette-Guérin (BCG) adjuvant was produced in Venezuela and utilized as an immunotherapy approach in patients with CL. Prophylactic investigations of this vaccine remained unresolved as acceptable results were not obtained. Furthermore, trivalent preparation including L. braziliensis, L. amazonensis, and Leishmania guyanensis were investigated in Ecuador. The results will be described with more details..$^{95,96}$

\section{Brazil}

Brazilian researchers have performed some trials with different preparations of killed parasites. Three doses of a polyvalent vaccine made of 18 strains of Leishmania were 
administrated. In this trial, the number of healthy volunteers was 1,127 and results were obtained with $80 \%$ efficacy. These were done by Mayrink et al to develop a pentavalent vaccine program in the 1970s. Unfortunately, the efficacy of the vaccine was not evaluated in this trial. ${ }^{94-98}$

In 1981 and 1983, two double-blind randomized control trials of pentavalent vaccine with the use of various doses have been conducted in Brazilian army personnel. In 1981, the results of this experiment did not show any remarkable differences between placebo groups and the vaccine group. In 1983, more than 1,200 army personnel were randomly recruited to the vaccine and placebo groups. The results of this assay did not show any significant differences between two groups as well. Unlike promising results, the leishmania vaccine was not broadly used because of difficulty in its production and standardization, as mentioned above. Moreover, further studies showed that the preparation of the single strain L. amazonensis vaccine refers to the same immunogenicity, and that utilization of the single strain L. amazonensis (IFA/BR/67/PH8) alone to vaccination was adopted. It is well known worldwide that the vaccine containing this strain can stimulate immune responses in vaccinated volunteers since it includes killed promastigotes and each dose contains $250 \mu \mathrm{g}$ of Corynebacterium parvum and $100 \mu \mathrm{g}$ of Leishmania protein. Different concentrations of monovalent vaccine in two to three doses of the vaccine could be confirmed in human volunteers. Of course, these results are found in separate studies. ${ }^{94,97,99-101}$

\section{Colombia}

The safety and immunogenicity of three doses of the monovalent L. amazonensis vaccine were approved in Colombia. In Phase II trial of vaccination, 296 army volunteers were injected intradermal and BCG as an adjuvant. Vaccination was also conducted intramuscularly without BCG. The perceived results of this clinical trial study indicated that this vaccination was immunogenic and safe, but did not provide protection. ${ }^{102,103}$

\section{Ecuador}

Only high-efficacy leishmaniasis vaccine was observed in Ecuador. In this study, efficacy, safety, and immunogenicity of trivalent vaccine were prepared, and intradermal doses of this vaccine were evaluated. The vaccine was made of L. braziliensis, L. amazonensis, and L. guyanensis promastigotes. This study included 438 individuals in case and 406 individuals in control groups. Lesions of patients living in the study areas were collected and mixed with BCG. In the study, the follow-up of patients 12 months after receiving vaccines indicated that utilization of this vaccine was safe. ${ }^{94,104,105}$

\section{Iran}

Following the cessation of the leishmanization plan in the 1980s, the Iranian government designed a vaccine development program to extend the killed leishmaniasis vaccine at the Razi serum institute. ${ }^{106,107}$

Phase I and II studies of clinical trials investigated immunogenicity and safety of different doses of inactivated L. major promastigotes with or without BCG and without adjuvants in Iran's non-endemic areas. The results of this study showed that reduced dose of BCG could have robust immune responses to antigens. The vaccines were produced by two methods for deactivation of parasites, that is, autoclaving versus thimerosal treatment, which demonstrated the same results for safety and immunogenicity due to their simplicity and reproducibility. But autoclaving was recommended as the preferred method for vaccine preparation in future trials. In this regard, studies with autoclaved L. major mixed with BCG in several trials were performed in Iran and Sudan. The results of some of these studies in the late 1990s have not been published yet. This preparation was replaced by a formulation comprising the precipitation of the autoclaved L. major in alum. However, autoclaved L. major-alum mixed with BCG as an adjuvant has been utilized only in Phase I and II clinical trials. The results of this study showed that immunogenicity, safety, and efficacy factors were acceptable in endemic areas of Esfahan, but that was unsuccessful because there were many problems with the leishmanization reagent. ${ }^{94,108-110}$

\section{Sudan}

Immunogenicity and safety autoclaved L. major mixed with BCG as an adjuvant was assessed in endemic and nonendemic areas in Sudan. Consequently, double-blind randomized clinical trials of two doses of autoclaved L. major with $\mathrm{BCG}$, in comparison with BCG alone, have been designed in endemic areas for VL, which was created by L. donovani. After 2 years' follow-up, the results did not show significant differences between the two groups. Besides these studies, autoclaved L. major with alum mixed with BCG was appraised for immunotherapeutic effects on patients with chronic post-kala-azar dermal leishmaniasis (PKDL) based on a clinical trial in Sudan and showed promising results in this respect. ${ }^{83,111-113}$ 
Based on the discussion about first-generation prophylactic vaccine, we found that the efficacy of these vaccines due to the use of killed whole parasites could not generate longlasting immunity, and the point of economic view is not reproducible. However, the results of the first-generation vaccine indicated the safety profile of these vaccines, and immunotherapy with the first-generation vaccine plus antimony drug has been registered in Brazil. Hence, immunotherapy with the first-generation vaccine could not be an acceptable therapeutic option. In this regard, we will discuss combination therapy with more details in following sections. To produce the first-generation vaccine, a 10 - to 12 -year period is required with a total cost of US\$2-3 million in Iran, Sudan, Ecuador, and Colombia. ${ }^{94}$

\section{Second-generation vaccine}

\section{Live vaccines}

This classification includes genetically modified vaccines in which essential genes such as thymidylate synthase, dihydrofolate reductase, cysteine proteinase, and/or biopterin transporter were knocked out. These parasites can generate adaptive immune responses adequately, resulting in inactivated infection and subsequently, disease does not occur in vaccinated people. Another approach, suicidal cassettes, is suggested in the Leishmania genome. Suicidal cassettes consist of drug-sensitive genes such as cytosine deaminase gene of Saccharomyces cerevisiae that is sensitive to 5 -fluorocytosine, or the expression of thymidine kinase gene of Herpes Simplex virus I that is sensitive to ganciclovir. Use of parasites with suicidal cassettes provides situations that would be able to guarantee effective treatment of incurable lesions and infections resistant to current chemotherapy. But due to ethical problems, utilization of live challenge in therapeutic approaches for humans has not been approved. However, many studies show that immune responses against live pathogens containing special antigens would be able to make long-lasting immunity against leishmaniasis. ${ }^{114-121}$

\section{Leishmania-extracted vaccines}

The first designed vaccine for canine VL, which consisted of purified $L$. donovani, is called Leishmune. Fructose mannose ligand (FML) and saponins as an adjuvant were utilized for the generation of this vaccine. L. donovani creates three main antigenic complexes and NH36 is a major antigen in this complex, which involves constructing parasite's DNA. Leishmune is a promising option to prevent canine VL. This vaccine is considered to suppress the transmission of zoonotic VL. Owing to its best immunoprotective role, purified Leishmania extract is widely used in experimental models. There are several difficulties to design vaccine in clinical trials such as large scale, standardization, in vitro culture condition. ${ }^{122,123}$

\section{Recombinant bacteria and viruses as delivery vehicles in vaccination}

Utilization of live recombinant bacteria or virus expressing Leishmania antigen is another strategy for the preparation of second-generation vaccine. In fact, bacteria or virus acts as an expression vehicle and an adjuvant system. In this regard, bacterial vaccines where developed by cloning the GP63 surface protease of $L$. major antigen in S. Thyphimurium mutant, or BCG.KMB-11 antigen in attenuated tachyzoites of Toxoplasma gondii and LCR1 antigen of L. chagasi antigen in BCG. Along with this, some vaccines were designed based on virus, such as virus expressing the LACK antigen of $L$. infantum, which protected mice against $L$. major via robust vaccination, and virus expressing promastigote protein surface of G46/M-2/PSA-2, which protected against L. amazonensis. Furthermore, promising effects of this strategy have confirmed that CD4- and CD8-specific immune responses could be reinforced through immunization process with increased IL-2, IFN- $\gamma$, and TNF- $\alpha$ in vaccinated groups. Therefore, by recruiting the new approaches in recombinant technology and improving the formulation a better purified vaccine could be created by increasing the stability, reproducibility, and safety of the antigens. This approach could reduce harmful reactions. In addition, administration of major immunogenic proteins provides path to generate integrated immune responses, especially against specified protective antigens. ${ }^{124}$

As a result, in this approach, despite the benefits of recombinant vaccine in a delivery system, special attention should be paid to high immunogenicity and cytotoxicity before administration. ${ }^{125-129}$

\section{Vaccines based on purified leishmania antigens}

It is thought that the main aim of the second-generation vaccine was to involve purified leishmania to their extracts. In this regard, lipophosphoglycan (LPG) and proteins have been used to evaluate the immunogenicity. In fact, FML is considered for developing a second-generation vaccine, which is based on the Gp36 glycoprotein of $L$. donovani fractions antigenicity effects and a mixture of FML and a saponin-derived plant as an adjuvant. This vaccine could be licensed as Leishman in Brazil to vaccinate dogs. Clinical trials have shown that administration of this vaccine is 
performed three times once every 21 days subcutaneously. Adverse effects of Leishman vaccine are tolerable and because of promotion in Th1 immune responses an increase of IFN- $\gamma$ cytokine could represent a suitable index of humoral and cellular immunity. Also, studies have indicated that this vaccine when mixed with Quillaja saponaria saponin as an adjuvant presents a safe and effective function in vaccination. But unfortunately, this approach could not be taken to Phase II or Phase III because of some difficulties that existed in the production way. ${ }^{128-133}$ Results of mode of action of FML-QS21 indicate that bradykinin activity could mediate inflammatory responses at the site of injection which stimulate immature dendritic cells, and consequently, this mechanism will result in triggering Th1 immune responses against leishmaniasis, especially L. chagasi. ${ }^{130-132}$

\section{Recombinant antigen}

Use of recombinant proteins is the last strategy in the second-generation vaccine, which was assayed from the 1990s. Recombinant vaccine candidates for leishmaniasis in combination, alone, or as chimeras poly-protein were evaluated. To enhance protection, many of them must be formulated with adjuvant or transmitted by bacteria. Only a few of these vaccines could be advanced to studies of preclinical trials in humans.

\section{Various recombinant antigens}

Recombinant vaccine candidates present induction of protective immunity by poly-protein vaccine formulation. Also, TSA (thiol-specific antioxidant) and LmSTI1 (L. major stress-inducible protein 1) are protective against CL in mice and monkeys. IL-12, as a recombinant adjuvant, is not recommended due to problems that are related to immune disorders. Other studies have shown that the leish-111f MPL fusion protein containing antigens LmTSTI1, TSA, and LeIF (Leishmania elongation initiation factor) in formulation with squalene and MPL-SE9 could protect mice against VL and CL. But further studies in healthy volunteers who received Leish111f plus MPL-SE showed to be safe and immunogenic in the USA and in individuals with CL and ML in Brazil and Peru. In this regard, six individuals with MCL treated with a combination therapy performed with Leish111f plus leishmania heat shock protein 83 (Lbhsp83) and GM-CSF showed improvement. Finally, poloxamer407 adjuvant $\mathrm{CPb}$ could protect mice against CL, while its combination with QuilA and IL-12 did not prove successful in protecting dogs against $L$. infantum. This weak protection may be due to low concentration of adjuvants $(50 \mu \mathrm{g}$ doses in each vaccine). ${ }^{132-138}$ Thus, there are many proteins and antigens to formulate vaccines to produce immune responses against leishmaniasis. However, many studies remain in the clinical trial level to determine their efficacies.

\section{New recombination antigen from other species}

Nonpathogenic leishmania has been isolated from a reptile animal called Leishmania tarentolae, which could not cause an infection in humans. So, various approaches have been focused to convert this leishmania into a vehicle for delivering antigens to the immune system. In this respect, Breton et al reported that administration with $L$. tarentolae as a vaccine can protect BALB/C mice against $L$. donovani. Also, other studies worked on A2 gene as responsible for visceral nature of $L$. donovani and $L$. infantum that were transmitted into L. tarentolae and utilized in BALB/C mice against $L$. donovani and $L$. infantum. The results of this assay provided protective immune responses with elevated IFN- $\gamma$ and reduced IL-5. This result is compatible with Breton's study in this field and also with the index of quality immunity in vaccination with $L$. tarentolae. ${ }^{16,139,140}$ Thus, it is concluded that the use of the new strategy in vaccination with $L$. tarentolae as a new vehicle could generate immune responses to provide protection against leishmaniasis. But more studies are needed to clarify which kind of immunity is more involved in this infection.

\section{Third-generation vaccine}

Studies have shown that DNA vaccines are much more stable than recombinant protein vaccines and they also have a lower cost of production compared with other vaccines. Along with this, distribution does not need a cold chain and there is considerable flexibility in combining several different genes into a product. The mode of action in DNA vaccines is performed by generation of immune responses through activation of innate immunity, which, in sequences of nonmethylated $\mathrm{CpG}$ of bacteria, are engaged and high replication within the host could lead to expression of the recombinant proteins for a longer period and with native conformation. ${ }^{132,141}$ Most of the antigens mentioned in the previous section were assayed as single vaccines, while some of them were evaluated as a combination of genes or heterologous prime-boost which include administration of the DNA vaccine, followed by administration of recombinant proteins. Vaccination with plasmids showed a protective role. However, no data on these vaccines are available in Phase III. Mice vaccination with TSA or LmSTI1 DNA vaccines can protect against CL by a $\mathrm{CD}^{+}$Th1 response. The results of the studies demonstrated 
that the TSA gene was the most protective vaccine with the later including a $\mathrm{CD}^{+}$response. ${ }^{132,142-146}$ DNA vaccine studies were performed in clinical trial Phases I and II and animals that were tested included dogs and mice. However, these promising results should be carefully considered and further confirmed by subsequent studies.

As a result, the second generation with native antigens could increase average vaccine efficacy value remarkably. Therefore, more studies and clinical trial in Phase III will be conducted in near future.

\section{New approaches in vaccination}

\section{Cationic solid lipid nanoparticle to deliver antigen}

Recently, new studies have focused on a delivery system called cationic solid lipid nanoparticle (cSLN). This is the carrier of the nanolipids to deliver vaccines and drugs. The main properties of the cSLN are its stability, resistance to oxidation, and no specific requirement for packaging. cSLN has more chemical stability and several surfactants than liposomes and utilizes lipids in their design. The cSLN functions to deliver antigens in the following ways: 1) absorption on the surface through electrical interaction with cationic cSLN and 2) the lipid matrix of the SLN, which could trap encapsulated antigens inside it. In this regard, Saljoughian et al worked on a DNA vaccine containing the $L$. donovani A2 gene besides L. infantum cysteine proteinase. The results of this study showed that injections of pCDNA-A2-CPA$\mathrm{CPB}^{-\mathrm{CTE}}$, delivered by cSLN, could protect $\mathrm{Balb} / \mathrm{c}$ mice against $L$. infantum. The obtained results show increases in IFN- $\gamma$ and decreases in IL-10, thereby resulting in a robust induction of Th1 responses in leishmaniasis. Therefore, cSLN when used as a nanoparticle vehicle of Leishmania antigens could enhance protective immune responses and can be adopted as a high-efficacy strategy against VL. ${ }^{147-150}$

\section{Alginate as leishmania antigen delivery systems}

Another current approach is associated with alginate. Alginate is a linear polysaccharide and is soluble in water, as it contains $\beta$-D-mannuronic acid and $\alpha$-L-guluronic acid. This composition can cross-link into a solid matrix by adding di- or trivalent cations. Owing to biodegradability, safety, and low cost of this polymer, alginate would be a suitable adoption for the delivery system in the vaccine. Recent studies on alginate have demonstrated that this substance has potential characteristics to be an application in immunoadjuvant and delivery system. Along with this, utilization of the alginate microsphere containing antigenic proteins for animal immunization can induce humoral and cellular immunity. In this regard, Tafaghodi et al studied this delivery approach so that they could optimize the production method of the alginate microsphere in combination with $L$. major and QS adjuvant. The results of their study showed that the subcutaneous administration of alginate microspheres, in combination with $L$. major (ALM) with $\mathrm{CpG}$ ODNs, in Balb/c mice was able to induce cell-mediated immune responses and protect mice against leishmaniasis. ${ }^{116,151-153}$ As a result, alginate, as a delivery system, could perform with high efficacy due to its immunoadjuvant properties and induction of cellular immunity. However, this research will be continued and probably face obstacles in this respect.

\section{Immune-stimulating complexes and ISCOMATRIX to deliver leishmania antigen}

Immune-stimulating complexes (ISCOMs) are usually prepared by cholesterol, phospholipid, envelope protein antigen, and Quil A in Triton X100 incorporated with different antigens, whereas ISCOMATRIX is an adjuvant that can be prepared by a phospholipid, cholesterol, and saponins without any antigen. ISCOMATRIX vaccines were evaluated in clinical trials and indicated to be safe and induce robust antigen-specific humoral or cellular immune responses against a wide spectrum of antigens. Sjo Lander et al studied the vaccination of PSA-2 DNA in Balb/c mice, which gave protection against $L$. major infection and increased Th1 immune responses predominantly. On the contrary, PSA2 ISCOMs generate a mixed immune response of Th1 and Th2 types. It was concluded that PSA-2 purified from Escherichia coli and injected into ISCOMs or with $C$. parvum as an adjuvant can induce Th1 responses intensely; however, it cannot provide protection. Papadopoulou et al demonstrated that peritoneal injections to vaccinate Balb/c mice with low doses of gp63 into ISCOMs can enhance partial protection against L. major and switch to Th1-type immune responses. ${ }^{16,154}$ As a result, immune stimulatory and delivery lipid systems have adequate potential to solve limitation problems and present obstacles in leishmaniasis treatment. One of the important applications of these special carriers is to avoid unsuitable degradation antigens and drugs from humoral proteolysis. This mechanism can play a significant role in generating central memory responses against leishmaniasis. According to the abovementioned benefits, there are many concerns about the therapeutic approach due to its toxicity and drug resistance. Thus, standardization and optimization of formulation parameters would be performed to promote adjuvants and delivery properties against leishmaniasis. ${ }^{155}$ 


\section{Evasion mechanisms of leishmania against treatment}

Protozoan parasites of the Leishmania gene are responsible for a wide range of diseases termed as leishmaniasis. Currently, efforts are being made to find new targets in therapeutic options. Two surface molecules of LPG and GP63 could inhibit the macrophage proteolytic function. LPG can elevate promastigotes intracellular survival by inhibiting the fusion of the phagosome with the lysosome in infected macrophages with $L$. donovani during the initial stages of infection. GP63 could prevent the phagolysosome enzyme function even if it gets formed in macrophages. Along with this, LPG and GP63 have a role in inhibiting oxidative bursts through the reduction of PKC translocation to the membrane. Also, the parasite enhances its survival by preventing apoptosis and by the presence of antigen presentation in macrophages. Apoptosis inhibition could happen via the stimulation of GM-CSF and TNF- $\alpha$. In this regard, GP63 of $L$. donovani and L. major cleave $\mathrm{CD}^{+}$on $\mathrm{T}$ cells and distribute interactions between cells where antigens are present on T cells. Moreover, amastigotes degrade MHCII and decrease B7.1. Along with secretion of TGF- $\beta$ and PGE2, this could lead to inhibition of macrophage function. LPG also decreases the TNF- $\alpha$ receptor expression and inhibits neutrophils and macrophages. Metacyclic promastigotes have a role to challenge complement components obtained through LPG elongation on their surface. GLP63 could block the proteolysis activity of $\mathrm{C} 3 \mathrm{~b}$ to iC $3 \mathrm{~b}$. Finally, Leishmania protein kinases can phosphorylate some of the complement components, including $\mathrm{C} 3, \mathrm{C} 5$, and $\mathrm{C} 9$, and inhibit classic and alternative pathways. ${ }^{156}$ As a result, parasites, especially Leishmania, can evade immune responses with various mechanisms. In addition, these mechanisms can help to maintain its survival and live compatibly with host circumstances. Hence, parasites can persist within immune cells, and this may contribute to the prevalence of drug resistance. Based on these findings, novel therapeutic tools should be designed to overcome this resistance.

The abovementioned concept could be explained by the following points:

Development of safe and effective vaccines would be possible for the following reasons:

- There is no significant antigenic variation in Leishmania.

- Macrophage as a single host cell for Leishmania is considered.

- Amastigote is a single morphological form that is associated with the pathology in the mammalian host.
- Reliable adjuvants that could induce cellular immunity, such as Th1 response, are now available.

These indicate that access to a perfect vaccine may eradicate leishmaniasis. But, such a perfect vaccine is yet to be designed. ${ }^{157}$

\section{Conclusion}

Finally, it is believed that controlling the sand fly vector for the sake of public health control and elimination of leishmaniasis is a major approach in this respect - this, in fact, can eradicate smallpox and remarkably decrease mortality and morbidity related to a variety of infectious diseases. Meanwhile, as mentioned in this review article, combination therapy of drugs and vaccines can successfully treat patients with leishmaniasis. However, adversary side effects and the high cost of treatment are the most common challenges in this respect.

In a comparison between vaccines and drugs, it should be noted that vaccines are the best affordable way to combat infections. Immunization with vaccines can save billions of people annually. Thus, more intense efforts are needed to produce preventive therapeutic vaccines. Although drug therapy almost immediately renders infections inactive, vaccination does not only give long-term protection against a disease but also works on infectious reservoirs to reduce transmission. In reality, a drug does not need to be designed, provided that a safe and perfect vaccine is made available. It is to be noted that there are some vaccines that can elicit long-term protection against leishmaniasis, but they are yet to be tested on humans through the clinical trials. Future trails are needed to resolve this and obtain an absolute vaccine for Leishmania infections.

Hence, we conclude that existing drug therapy or vaccination therapy is not a perfect therapeutic way to eradicate leishmaniasis. More researches are needed to prepare an effective therapeutic vaccine.

\section{Acknowledgment}

We would like to appreciate Dr Davoud Nouri Inanlou for his kind and generous help in this review.

\section{Disclosure}

The authors report no conflicts of interest in this work.

\section{References}

1. Cabezas Y, Legentil L, Robert-Gangneux F, et al. Leishmania cell wall as a potent target for antiparasitic drugs. A focus on the glycoconjugates. Org Biomol Chem. 2015;13(31):8393-8404. 
2. de Freitas EO, Leoratti FM, Freire-de-Lima CG, Morrot A, Feijó DF. The contribution of immune evasive mechanisms to parasite persistence in visceral leishmaniasis. Front Immunol. 2016;7:153.

3. de Vries HJ, Reedijk SH, Schallig HD. Cutaneous leishmaniasis: recent developments in diagnosis and management. Am J Clin Dermatol. 2015;16(2):99-109.

4. Pearson RD, Sousa AQ. Clinical spectrum of leishmaniasis. Clin Infect Dis. 1996:1-11.

5. Savoia D. Recent updates and perspectives on leishmaniasis. J Infect Dev Ctries. 2015;9(6):588-596.

6. Moll H, Berberich C. Dendritic cell-based vaccination strategies: induction of protective immunity against leishmaniasis. Immunobiology. 2001;204(5):659-666.

7. Peters NC, Egen JG, Secundino N, et al. In vivo imaging reveals an essential role for neutrophils in leishmaniasis transmitted by sand flies. Science. 2008;321(5891):970-974.

8. Amulic B, Cazalet C, Hayes GL, Metzler KD, Zychlinsky A. Neutrophil function: from mechanisms to disease. Annu Rev Immunol. 2012; 30:459-489.

9. Antonelli LR, Dutra WO, Almeida RP, Bacellar O, Carvalho EM, Gollob KJ. Activated inflammatory T cells correlate with lesion size in human cutaneous leishmaniasis. Immunol Lett. 2005;101(2): 226-230.

10. Bacellar O, Lessa H, Schriefer A, et al. Up-regulation of Th1-type responses in mucosal leishmaniasis patients. Infect Immun. 2002;70(12): 6734-6740.

11. Conceição J, Davis R, Carneiro PP, et al. Characterization of neutrophil function in human cutaneous leishmaniasis caused by leishmania braziliensis. PLoS Negl Trop Dis. 2016;10(5):e0004715.

12. Müller K, van Zandbergen G, Hansen B, et al. Chemokines, natural killer cells and granulocytes in the early course of Leishmania major infection in mice. Med Microbiol Immunol. 2001;190(1-2):73-76.

13. Ribeiro-de-Jesus A, Almeida RP, Lessa H, Bacellar O, Carvalho EM. Cytokine profile and pathology in human leishmaniasis. Braz J Med Biol Res. 1998;31(1):143-148.

14. Freitas-Junior LH, Chatelain E, Kim HA, Siqueira-Neto JL. Visceral leishmaniasis treatment: what do we have, what do we need and how to deliver it? Int J Parasitol Drug Resist. 2012;2:11-19.

15. Rajasekaran R, Chen YP. Potential therapeutic targets and the role of technology in developing novel antileishmanial drugs. Drug Discov Today. 2015;20(8):958-968.

16. Duthie MS, Favila M, Hofmeyer KA, et al. Strategic evaluation of vaccine candidate antigens for the prevention of visceral leishmaniasis Vaccine. 2016;34(25):2779-2786.

17. Sundar S. Drug resistance in Indian visceral leishmaniasis. Trop Med Int Health. 2001;6(11):849-854.

18. Croft SL, Coombs GH. Leishmaniasis-current chemotherapy and recent advances in the search for novel drugs. Trends Parasitol. 2003 19(11):502-508

19. Dorlo TP, Rijal S, Ostyn B, et al. Failure of miltefosine in visceral leishmaniasis is associated with low drug exposure. J Infect Dis. 2014; 210(1):146-153.

20. Jain K, Jain NK. Vaccines for visceral leishmaniasis: a review. J Immunol Methods. 2015;422:1-12.

21. Minodier P, Parola P. Cutaneous leishmaniasis treatment. Travel Med Infect Dis. 2007;5(3):150-158.

22. Moskowitz PF, Kurban AK. Treatment of cutaneous leishmaniasis: retrospectives and advances for the 21st century. Clin Dermatol. 1999 17(3):305-315.

23. Reithinger R, Dujardin JC, Louzir H, Pirmez C, Alexander B, Brooker S. Cutaneous leishmaniasis. Lancet Infect Dis. 2007;7(9):581-596.

24. Palumbo E. Treatment strategies for mucocutaneous leishmaniasis. J Glob Infect Dis. 2010;2(2):147-150.

25. Aste N, Pau M, Ferreli C, Biggio P. Intralesional treatment of cutaneous leishmaniasis with meglumine antimoniate. Br J Dermatol. 1998; 138(2):370-371
26. Blum J, Desjeux P, Schwartz E, Beck B, Hatz C. Treatment of cutaneous leishmaniasis among travellers. J Antimicrob Chemother. 2004;53(2): 158-166.

27. Manfredi M, Iuliano S, Bizzarri B, Fugazza A, Gismondi P. Cutaneous leishmaniasis with long duration and bleeding ulcer. Clin Microbiol. 2016;5:229.

28. Coelho DR, De-Oliveira A, Parente T, et al. In vivo and in vitro effects of pentavalent antimony on mouse liver cytochrome P450s. Hum Exp Toxicol. Epub 2016 Mar 4.

29. Herman JD, Gallalee JV, Best JM. Sodium stibogluconate (Pentostam) inhibition of glucose catabolism via the glycolytic pathway, and fatty acid $\beta$-oxidation in Leishmania mexicana amastigotes. Biochem Pharmacol. 1987;36(2):197-201.

30. Croft SL, Sundar S, Fairlamb AH. Drug resistance in leishmaniasis. Clin Microbiol Rev. 2006;19(1):111-126.

31. de Souza Moreira D, Ferreira RF, Murta SM. Molecular characterization and functional analysis of pteridine reductase in wild-type and antimony-resistant Leishmania lines. Exp Parasitol. 2016;160: $60-66$

32. Nare B, Garraway LA, Vickers TJ, Beverley SM. PTR1-dependent synthesis of tetrahydrobiopterin contributes to oxidant susceptibility in the trypanosomatid protozoan parasite Leishmania major. Curr Genet. 2009;55(3):287-299.

33. de Moura TR, Santos ML, Braz JM, et al. Cross-resistance of Leishmania infantum isolates to nitric oxide from patients refractory to antimony treatment, and greater tolerance to antileishmanial responses by macrophages. Parasitol Res. 2016;115(2):713-721.

34. Adler-Moore JP, Proffitt RT. Development, characterization, efficacy and mode of action of AmBisome, a unilamellar liposomal formulation of amphotericin B. J Liposome Res. 1993;3(3):429-450.

35. Al-Abdely HM, Graybill JR, Bocanegra R, et al. Efficacies of KY62 against Leishmania amazonensis and Leishmania donovani in experimental murine cutaneous leishmaniasis and visceral leishmaniasis. Antimicrob Agents Chemother. 1998;42(10):2542-2548.

36. Espuelas MS, Legrand P, Campanero MA, et al. Polymeric carriers for amphotericin B: in vitro activity, toxicity and therapeutic efficacy against systemic candidiasis in neutropenic mice. $J$ Antimicrob Chemother. 2003;52(3):419-427.

37. Guo LS, Working PK. Complexes of amphotericin B and cholesteryl sulfate. J Liposome Res. 1993;3(3):473-490.

38. Larabi M, Gulik A, Dedieu JP, Legrand P, Barratt G, Cheron M. New lipid formulation of amphotericin B: spectral and microscopic analysis. Biochim Biophys Acta. 2004;1664(2):172-181.

39. Lopez-Berestein G, Hopfer RL, Mehta R, Mehta K, Hersh EM, Juliano RL. Liposome-encapsulated amphotericin B for treatment of disseminated candidiasis in neutropenic mice. J Infect Dis. 1984; 150(2):278-283.

40. Stone NR, Bicanic T, Salim R, Hope W. Liposomal amphotericin B $\left(\right.$ AmBisome $\left.{ }^{\circledR}\right)$ : a review of the pharmacokinetics, pharmacodynamics, clinical experience and future directions. Drugs. 2016;76(4):485-500.

41. Adler-Moore J, Proffitt RT. AmBisome: liposomal formulation, structure, mechanism of action and pre-clinical experience. $J$ Antimicrob Chemother. 2002;49(suppl 1):21-30.

42. Fujii G, Chang JE, Coley T, Steere B. The formation of amphotericin B ion channels in lipid bilayers. Biochemistry. 1997;36(16):4959-4968.

43. Papahadjopoulos D, Jacobson K, Nir S, Isac I. Phase transitions in phospholipid vesicles fluorescence polarization and permeability measurements concerning the effect of temperature and cholesterol Biochim Biophys Acta. 1973;311(3):330-348.

44. Wasan EK, Gershkovich P, Zhao J, et al. A novel tropically stable oral amphotericin B formulation (iCo-010) exhibits efficacy against visceral Leishmaniasis in a murine model. PLoS Negl Trop Dis. 2010; 4(12):e913.

45. Mistro S, Rodrigues M, Rosa L, Camargo M, Badaró R. Liposomal amphotericin B drug access for the treatment of leishmaniasis in Brazil. Trop Med Int Health. 2016;21(6):692-693. 
46. Bellmann R. Clinical pharmacokinetics of systemically administered antimycotics. Curr Clin Pharmacol. 2007;2(1):37-58.

47. Bellmann R, Egger P, Wiedermann CJ. Differences in pharmacokinetics of amphotericin B lipid formulations despite clinical equivalence. Clin Infect Dis. 2003;36(11):1500-1501.

48. Felton T, Troke PF, Hope WW. Tissue penetration of antifungal agents. Clin Microbiol Rev. 2014;27(1):68-88.

49. Groll AH, Lyman CA, Petraitis V, et al. Compartmentalized intrapulmonary pharmacokinetics of amphotericin B and its lipid formulations. Antimicrob Agents Chemother. 2006;50(10):3418-3423.

50. Walsh TJ, Finberg RW, Arndt C, et al. Liposomal amphotericin B for empirical therapy in patients with persistent fever and neutropenia. N Engl J Med. 1999;340(10):764-771.

51. Croft SL. Monitoring drug resistance in leishmaniasis. Trop Med Int Health. 2001;6(11):899-905.

52. Mistro S, Maciel Ide M, de Menezes RG, Maia ZP, Schooley RT, Badaró R. Does lipid emulsion reduce amphotericin B nephrotoxicity? A systematic review and meta-analysis. Clin Infect Dis. 2012;54(12): 1774-1777.

53. Balasegaram M, Ritmeijer K, Lima MA, et al. Liposomal amphotericin $\mathrm{B}$ as a treatment for human leishmaniasis. Expert Opin Emerg Drugs. 2012;17(4):493-510.

54. Meheus F, Balasegaram M, Olliaro P, et al. Cost-effectiveness analysis of combination therapies for visceral leishmaniasis in the Indian subcontinent. PLoS Negl Trop Dis. 2010;4(9):e818.

55. Olliaro P, Darley S, Laxminarayan R, Sundar S. Cost-effectiveness projections of single and combination therapies for visceral leishmaniasis in Bihar, India. Trop Med Int Health. 2009;14(8):918-925.

56. Solomon M, Baum S, Barzilai A, Scope A, Trau H, Schwartz E. Liposomal amphotericin B in comparison to sodium stibogluconate for cutaneous infection due to Leishmania braziliensis. J Am Acad Dermatol. 2007;56(4):612-616.

57. Echevarría I, Barturen C, Renedo MJ, Trocóniz IF, Dios-Viéitez MC. Comparative pharmacokinetics, tissue distributions, and effects on renal function of novel polymeric formulations of amphotericin B and amphotericin B-deoxycholate in rats. Antimicrob Agents Chemother. 2000;44(4):898-904.

58. Fielding RM, Smith PC, Wang LH, Porter J, Guo LS. Comparative pharmacokinetics of amphotericin $\mathrm{B}$ after administration of a novel colloidal delivery system, $\mathrm{ABCD}$, and a conventional formulation to rats. Antimicrob Agents Chemother. 1991;35(6):1208-1213.

59. Khan MA, Owais M. Toxicity, stability and pharmacokinetics of amphotericin B in immunomodulator tuftsin-bearing liposomes in a murine model. J Antimicrob Chemother. 2006;58(1):125-132.

60. Wasan KM, Morton RE, Rosenblum MG, Lopez-Berestein G. Decreased toxicity of liposomal amphotericin B due to association of amphotericin B with high-density lipoproteins: Role of lipid transfer protein. J Pharm Sci. 1994;83(7):1006-1010.

61. Patere SN, Pathak PO, Kumar Shukla A, et al. Surface-modified liposomal formulation of amphotericin B: in vitro evaluation of potential against visceral leishmaniasis. AAPS Pharm Sci Tech. 2017;18(3): 710-720.

62. Singh PK, Sah P, Meher JG, et al. Macrophage-targeted chitosan anchored PLGA nanoparticles bearing doxorubicin and amphotericin B against visceral leishmaniasis. RSC Adv. 2016;6(75):71705-71718.

63. Larabi M, Legrand $P$, Barratt $G$. An original lipid complex system for amphotericin B. Liposome technology: entrapment of drugs and other materials into liposomes. Curr Opin Infect Dis. 2016;2:93.

64. Sundar S, Singh A. Recent developments and future prospects in the treatment of visceral leishmaniasis. Ther Adv Infect Dis. 2016;3(34): 98-109.

65. Brotherton MC, Bourassa S, Légaré D, Poirier GG, Droit A, Ouellette M. Quantitative proteomic analysis of amphotericin B resistance in Leishmania infantum. Int J Parasitol Drugs Drug Resist. 2014;4(2): 126-132.

66. Cohen BE. Resistance responses to amphotericin B: the role of signaling via aqueous pores formation. Antimicrob Agents Chemother. 2016; 60(9):5122-5129.
67. McGonigle S, Dalton JP, James ER. Peroxidoxins: a new antioxidant family. Parasitol Today. 1998;14(4):139-145.

68. Purkait B, Kumar A, Nandi N, et al. Mechanism of amphotericin B resistance in clinical isolates of Leishmania donovani. Antimicrob Agents Chemother. 2012;56(2):1031-1041.

69. Croft SL, Seifert K, Duchêne M. Antiprotozoal activities of phospholipid analogues. Mol Biochem Parasitol. 2003;126(2):165-172.

70. Dorlo TP, Balasegaram M, Beijnen JH, de Vries PJ. Miltefosine: a review of its pharmacology and therapeutic efficacy in the treatment of leishmaniasis. J Antimicrob Chemother. 2012;67(11):2576-2597.

71. Breiser A, Kim DJ, Fleer EA, et al. Distribution and metabolism of hexadecylphosphocholine in mice. Lipids. 1987;22(11):925-926.

72. Person C. Application for Inclusion of Emtricitabine On WHO Model List of Essential Medicines.Gilead Sciences, Inc. Foster City, California, USA, October 2004.

73. Ruiter GA, Zerp SF, Bartelink H, van Blitterswijk WJ, Verheij M. Anticancer alkyl-lysophospholipids inhibit the phosphatidylinositol 3-kinaseAkt/PKB survival pathway. Anticancer Drugs. 2003;14(2):167-173.

74. Coelho AC. Miltefosine susceptibility and resistance in leishmania: from the laboratory to the field. J Trop Dis. 2016;4:203.

75. Laffitte MC, Leprohon P, Légaré D, Ouellette M. Deep-sequencing revealing mutation dynamics in the miltefosine transporter gene in Leishmania infantum selected for miltefosine resistance. Parasitol Res. 2016;115(10):3699-3703.

76. Mondal D, Hasnain MG, Hossain MS, et al. Study on the safety and efficacy of miltefosine for the treatment of children and adolescents with post-kala-azar dermal leishmaniasis in Bangladesh, and an association of serum vitamin $\mathrm{E}$ and exposure to arsenic with post-kala-azar dermal leishmaniasis: an open clinical trial and case-control study protocol. BMJ Open. 2016;6(5):e010050.

77. Pérez-Victoria FJ, Gamarro F, Ouellette M, Castanys S. Functional cloning of the miltefosine transporter A novel P-type phospholipid translocase from leishmania involved in drug resistance. J Biol Chem. 2003;278(50):49965-49971.

78. Maran N, Gomes PS, Freire-de-Lima L, Freitas EO, Freire-de-Lima CG, Morrot A. Host resistance to visceral leishmaniasis: prevalence and prevention. Expert Rev Anti Infect Ther. 2016;14(4):435-442.

79. Wasunna M, Njenga S, Balasegaram M, et al. Efficacy and safety of AmBisome in combination with sodium stibogluconate or Miltefosine and Miltefosine Monotherapy for African Visceral Leishmaniasis: phase II randomized trial. PLoS Negl Trop Dis. 2016;10(9):e0004880.

80. McMichael AJ, Koff WC. Vaccines that stimulate T cell immunity to HIV-1: the next step. Nat Immunol. 2014;15(4):319-322.

81. Choudhury R, Das P, De T, Chakraborti T. $115 \mathrm{kDa}$ serine protease confers sustained protection to visceral leishmaniasis caused by Leishmania donovani via IFN- $\gamma$ induced down-regulation of TNF- $\alpha$ mediated MMP-9 activity. Immunobiology. 2013;218(1):114-126.

82. Giunchetti RC, Reis AB, da Silveira-Lemos D, et al. Antigenicity of a whole parasite vaccine as promising candidate against canine leishmaniasis. Res Vet Sci. 2008;85(1):106-112.

83. Khalil EA, El Hassan AM, Zijlstra EE, et al. Autoclaved Leishmania major vaccine for prevention of visceral leishmaniasis: a randomised, double-blind, BCG-controlled trial in Sudan. Lancet. 2000;356(9241):1565-1569.

84. Chakravarty J, Kumar S, Trivedi S, et al. A clinical trial to evaluate the safety and immunogenicity of the LEISH-F1+ MPL-SE vaccine for use in the prevention of visceral leishmaniasis. Vaccine. 2011;29(19):3531-3537.

85. Badaro R, Lobo I, Nakatani M, et al. Successful use of a defined antigen/ GM-CSF adjuvant vaccine to treat mucosal Leishmaniasis refractory to antimony: a case report. Braz J Infect Dis. 2001;5(4):223-232.

86. Genaro O, de Toledo VP, da Costa CA, Hermeto MV, Afonso LC, Mayrink W. Vaccine for prophylaxis and immunotherapy, Brazil. Clin Dermatol. 1996;14(5):503-512.

87. Mohebali M, Khamesipour A, Mobedi I, Zarei Z, Hashemi-Fesharki R. Double-blind randomized efficacy field trial of alum precipitated autoclaved Leishmania major vaccine mixed with BCG against canine visceral leishmaniasis in Meshkin-Shahr district, I.R. Iran. Vaccine. 2004;22(29-30):4097-4100. 
88. Noazin S, Khamesipour A, Moulton LH, et al. Efficacy of killed wholeparasite vaccines in the prevention of leishmaniasis: a meta-analysis. Vaccine. 2009;27(35):4747-4753.

89. Duthie MS, Reed SG. The emergence of defined subunit vaccines for the prevention of leishmaniasis. Curr Trop Med Rep. 2014;1(3):154-162.

90. Guha R, Das S, Ghosh J, et al. Heterologous priming-boosting with DNA and vaccinia virus expressing kinetoplastid membrane protein-11 induces potent cellular immune response and confers protection against infection with antimony resistant and sensitive strains of Leishmania (Leishmania) donovani. Vaccine. 2013;31(15):1905-1915.

91. Guha R, Gupta D, Rastogi R, et al. Vaccination with leishmania hemoglobin receptor-encoding DNA protects against visceral leishmaniasis. Sci Transl Med. 2013;5(202):202ra121.

92. Tewary P, Jain M, Sahani MH, Saxena S, Madhubala R. A heterologous prime-boost vaccination regimen using ORFF DNA and recombinant ORFF protein confers protective immunity against experimental visceral leishmaniasis. J Infect Dis. 2005;191(12):2130-2137.

93. Mahdavi M, Mavandadnejad F, Yazdi MH, et al. Oral administration of synthetic selenium nanoparticles induced robust Th1 cytokine pattern after HBs antigen vaccination in mouse model. J Infect Public Health. 2017;10(1):102-109.

94. Noazin S, Modabber F, Khamesipour A, et al. First generation leishmaniasis vaccines: a review of field efficacy trials. Vaccine. 2008;26(52):6759-6767.

95. Armijos RX, Weigel MM, Aviles H, Maldonado R, Racines J. Field trial of a vaccine against new world cutaneous leishmaniasis in an at-risk child population: safety, immunogenicity, and efficacy during the first 12 months of follow-up. J Infect Dis. 1998;177(5): 1352-1357.

96. Velez I, Agudelo S, Hendrickx E, et al. Inefficacy of allopurinol as monotherapy for Colombian cutaneous leishmaniasis: a randomized, controlled trial. Ann Intern Med. 1997;126(3):232-236.

97. Machado-Pinto J, Pinto J, da Costa CA, et al. Immunochemotherapy for cutaneous leishmaniasis: a controlled trial using killed Leishmania (Leishmania) amazonensis vaccine plus antimonial. Int J Dermatol. 2002;41(2):73-78.

98. Mayrink W, da Costa CA, Magalhães PA, et al. A field trial of a vaccine against American dermal leishmaniasis. Trans $R$ Soc Trop Med Hyg. 1979;73(4):385-387.

99. Antunes CM, Mayrink W, Magalhaes PA, et al. Controlled field trials of a vaccine against new world cutaneous leishmaniasis. Int $J$ Epidemiol. 1986;15(4):572-580.

100. De Luca PM, Mayrink W, Pinto JA, et al. A randomized double-blind placebo-controlled trial to evaluate the immunogenicity of a candidate vaccine against American tegumentary leishmaniasis. Acta Trop. 2001;80(3):251-260.

101. Mayrink W, Santos GC, Toledo Vde P, et al. Vaccination of C57BL/10 mice against cutaneous leishmaniasis using killed promastigotes of different strains and species of Leishmania. Rev Soc Bras Med Trop. 2002;35(2):125-132.

102. Vélez ID, del Pilar Agudelo S, Arbelaez MP, et al. Safety and immunogenicity of a killed Leishmania (L.) amazonensis vaccine against cutaneous leishmaniasis in Colombia: a randomized controlled trial. Trans R Soc Trop Med Hyg. 2000;94(6):698-703.

103. Vélez ID, Gilchrist K, Arbelaez MP, et al. Failure of a killed Leishmania amazonensis vaccine against American cutaneous leishmaniasis in Colombia. Trans R Soc Trop Med Hyg. 2005;99(8):593-598.

104. Armijos RX, Weigel MM, Romero L, Garcia V, Salazar J. Field trial of a vaccine against new world cutaneous leishmaniasis in an at-risk child population: safety, immunogenicity, and efficacy during the first 12 months of follow-up. J Infect Dis. 1998;177(5):1352-1357.

105. Armijos RX, Weigel MM, Romero L, Garcia V, Salazar J. Field trial of a vaccine against new world cutaneous leishmaniasis in an at-risk child population: how long does protection last? J Infect Dis. 2003; 187(12):1959-1961.

106. Khamesipour A, Rafati S, Davoudi N, Maboudi F, Modabber F. Leishmaniasis vaccine candidates for development: a global overview. Indian J Med Res. 2006;123(3):423-438
107. Modabber F. First generation leishmaniasis vaccines in clinical development: moving, but what next. Curr Opin Investig Drugs. 2000;2: 35-39.

108. Mahmoodi M, Khamesipour A, Dowlati Y, et al. Immune response measured in human volunteers vaccinated with autoclaved Leishmania major vaccine mixed with low dose of BCG. Clin Exp Immunol. 2003;134(2):303-308.

109. Momeni AZ, Jalayer T, Emamjomeh M, et al. A randomised, doubleblind, controlled trial of a killed L. major vaccine plus BCG against zoonotic cutaneous leishmaniasis in Iran. Vaccine. 1999;17(5): 466-472.

110. Sharifi I, FeKri AR, Aflatonian MR, et al. Randomised vaccine trial of single dose of killed Leishmania major plus BCG against anthroponotic cutaneous leishmaniasis in Bam, Iran. Lancet. 1998; 351(9115):1540-1543.

111. Khalil EA, Elhassan AM, Zijlstra EE, et al. Safety and immunogenicity of an autoclaved Leishmania major vaccine. East Afr Med J. 2000;77(9):468-470.

112. Musa AM, Khalil EA, Mahgoub FA, et al; Leishmaniasis Research Group/Sudan. Immunochemotherapy of persistent post-kala-azar dermal leishmaniasis: a novel approach to treatment. Trans $R$ Soc Trop Med Hyg. 2008;102(1):58-63.

113. Satti IN, Osman HY, Daifalla NS, et al. Immunogenicity and safety of autoclaved Leishmania major plus BCG vaccine in healthy Sudanese volunteers. Vaccine. 2001;19(15-16):2100-2106.

114. Alexander J, Coombs GH, Mottram JC. Leishmania mexicana cysteine proteinase-deficient mutants have attenuated virulence for mice and potentiate a Th1 response. J Immunol. 1998;161(12):6794-6801.

115. Cruz A, Coburn CM, Beverley SM. Double targeted gene replacement for creating null mutants. Proc Natl Acad Sci U S A. 1991;88(16): $7170-7174$

116. Gholami E, Zahedifard F, Rafati S. Delivery systems for Leishmania vaccine development. Expert Rev Vaccines. 2016;15(7):879-895.

117. Muyombwe A, Olivier M, Harvie P, Bergeron MG, Ouellette M, Papadopoulou B. Protection against Leishmania major challenge infection in mice vaccinated with live recombinant parasites expressing a cytotoxic gene. J Infect Dis. 1998;177(1):188-195.

118. Edrissian GH, Mohammadi M, Kanani A, et al. Bacterial infections in suspected cutaneous leishmaniasis lesions. Bull World Health Organ. 1990;68(4):473-477.

119. Requena JM, Iborra S, Carrión J, Alonso C, Soto M. Recent advances in vaccines for leishmaniasis. Expert Opin Biol Ther. 2004;4(9): 1505-1517.

120. Schofield C. UNDP/World Bank/WHO special programme for research and training in tropical diseases (TDR). Parasitology Today. 1989;5(8):235-238.

121. Souza AE, Bates PA, Coombs GH, Mottram JC. Null mutants for the lmcpa cysteine proteinase gene in Leishmania mexicana. Mol Biochem Parasitol. 1994;63(2):213-220.

122. Afrin F, Rajesh R, Anam K, Gopinath M, Pal S, Ali N. Characterization of Leishmania donovani antigens encapsulated in liposomes that induce protective immunity in $\mathrm{BALB} / \mathrm{c}$ mice. Infect Immun. 2002;70(12):6697-6706.

123. Srivastava S, Shankar P, Mishra J, Singh S. Possibilities and challenges for developing a successful vaccine for leishmaniasis. Parasit Vectors. 2016;9(1):277.

124. Petitdidier E, Pagniez J, Papierok G, Vincendeau P, Lemesre JL, BrasGonçalves R. Recombinant forms of Leishmania amazonensis excreted/ secreted promastigote surface antigen (PSA) induce protective immune responses in dogs. PLoS Negl Trop Dis. 2016;10(5):e0004614.

125. Gonzalo RM, del Real G, Rodriguez JR, et al. A heterologous primeboost regime using DNA and recombinant vaccinia virus expressing the Leishmania infantum $\mathrm{P} 36 / \mathrm{LACK}$ antigen protects BALB/c mice from cutaneous leishmaniasis. Vaccine. 2002;20(7):1226-1231.

126. Ramírez JR, Gilchrist K, Robledo S, Sepúlveda JC, Moll H, Soldati D, et al. Attenuated Toxoplasma gondii ts- 4 mutants engineered to express the Leishmania antigen KMP-11 elicit a specific immune response in BALB/c mice. Vaccine. 2001;20(3):455-461. 
127. Ramiro MJ, Zárate JJ, Hanke T, et al. Protection in dogs against visceral leishmaniasis caused by Leishmania infantum is achieved by immunization with a heterologous prime-boost regime using DNA and vaccinia recombinant vectors expressing LACK. Vaccine. 2003;21(19-20):2474-2484.

128. Streit JA, Recker TJ, Donelson JE, Wilson ME. BCG expressing LCR1 of Leishmania chagasi induces protective immunity in susceptible mice. Exp Parasitol. 2000;94(1):33-41.

129. Xu D, Mcsorley SJ, Chatfield SN, Dougan G, Liew FY. Protection against Leishmania major infection in genetically susceptible BALB/c mice by gp63 delivered orally in attenuated Salmonella typhimurium (AroA-AroD-). Immunology. 1995;85(1):1-7.

130. Marciani DJ. Vaccine adjuvants: role and mechanisms of action in vaccine immunogenicity. Drug Discov Today. 2003;8(20):934-943.

131. Borja-Cabrera GP, Santos FN, Bauer FS, et al. Immunogenicity assay of the Leishmune vaccine against canine visceral leishmaniasis in Brazil. Vaccine. 2008;26(39):4991-4997.

132. Palatnik-de-Sousa CB. Vaccines for leishmaniasis in the fore coming 25 years. Vaccine. 2008;26(14):1709-1724.

133. Campos-Neto A, Porrozzi R, Greeson K, et al. Protection against cutaneous leishmaniasis induced by recombinant antigens in murine and nonhuman primate models of the human disease. Infect Immun. 2001;69(6):4103-4108.

134. Fujiwara RT, Vale AM, Franca da Silva JC, et al. Immunogenicity in dogs of three recombinant antigens (TSA, LeIF and LmSTI1) potential vaccine candidates for canine visceral leishmaniasis. Vet Res. 2005;36(5-6):827-838.

135. Ghalib H, Modabber F. Consultation meeting on the development of therapeutic vaccines for post kala azar dermal leishmaniasis. Kinetoplastid Biol Dis. 2007;6:7.

136. Gurunathan S, Prussin C, Sacks DL, Seder RA. Vaccine requirements for sustained cellular immunity to an intracellular parasitic infection. Nat Med. 1998;4(12):1409-1415.

137. Noormohammadi AH, Hochrein H, Curtis JM, Baldwin TM, Handman E. Paradoxical effects of IL-12 in leishmaniasis in the presence and absence of vaccinating antigen. Vaccine. 2001;19(28-29):4043-4052.

138. Poot J, Spreeuwenberg K, Sanderson SJ, et al. Vaccination with a preparation based on recombinant cysteine peptidases and canine IL-12 does not protect dogs from infection with Leishmania infantum. Vaccine. 2006;24(14):2460-2468.

139. Breton M, Tremblay MJ, Ouellette M, Papadopoulou B. Live nonpathogenic parasitic vector as a candidate vaccine against visceral leishmaniasis. Infect Immun. 2005;73(10):6372-6382.

140. Mizbani A, Taheri T, Zahedifard F, et al. Recombinant Leishmania tarentolae expressing the A2 virulence gene as a novel candidate vaccine against visceral leishmaniasis. Vaccine. 2009;28(1):53-62.

141. Mahdavi M, Ebtekar M, Khorram Khorshid HR, Azadmanesh K, Hartoonian C, Hassan ZM. ELISPOT analysis of a new CTL based DNA vaccine for HIV-1 using GM-CSF in DNA prime/peptide boost strategy: GM-CSF induced long-lived memory responses. Immunol Lett. 2011;140(1-2):14-20.

142. Campos-Neto A, Webb JR, Greeson K, Coler RN, Skeiky YA, Reed SG. Vaccination with plasmid DNA encoding TSA/LmSTI1 leishmanial fusion proteins confers protection against Leishmania major infection in susceptible BALB/c mice. Infect Immun. 2002;70(6):2828-2836.
143. Hartoonian C, Ebtekar M, Soleimanjahi H, et al. Effect of immunological adjuvants: GM-CSF (granulocyte-monocyte colony stimulating factor) and IL-23 (interleukin-23) on immune responses generated against hepatitis $\mathrm{C}$ virus core DNA vaccine. Cytokine. 2009;46(1):43-50.

144. Iborra S, Soto M, Carrión J, Alonso C, Requena JM. Vaccination with a plasmid DNA cocktail encoding the nucleosomal histones of Leishmania confers protection against murine cutaneous leishmaniosis. Vaccine. 2004;22(29-30):3865-3876.

145. Jamali A, Mahdavi M, Hassan ZM, et al. A novel adjuvant, the general opioid antagonist naloxone, elicits a robust cellular immune response for a DNA vaccine. Int Immunol. 2009;21(3):217-225.

146. Velashjerdi Farahani S, Reza Aghasadeghi M, Memarnejadian A, Faezi S, Shahosseini Z, Mahdavi M. Naloxone/alum mixture a potent adjuvant for HIV-1 vaccine: induction of cellular and poly-isotypic humoral immune responses. Pathog Glob Health. 2016;110(2):39-47.

147. Doroud D, Vatanara A, Zahedifard F, et al. Cationic solid lipid nanoparticles loaded by cysteine proteinase genes as a novel antileishmaniasis DNA vaccine delivery system: characterization and in vitro evaluations. J Pharm Pharm Sci. 2010;13(3):320-335.

148. Mehnert W, Mäder K. Solid lipid nanoparticles: production, characterization and applications. Adv Drug Deliv Rev. 2001;47(2-3):165-196.

149. MuÈller RH, MaÈder K, Gohla S. Solid lipid nanoparticles (SLN) for controlled drug delivery-a review of the state of the art. Eur J Pharm Biopharm. 2000;50(1):161-177.

150. Saljoughian N, Zahedifard F, Doroud D, et al. Cationic solid-lipid nanoparticles are as efficient as electroporation in DNA vaccination against visceral leishmaniasis in mice. Parasite Immunol. 2013;35(12):397-408.

151. Tafaghodi M, Eskandari M, Khamesipour A, Jaafari MR. Alginate microspheres encapsulated with autoclaved Leishmania major (ALM) and $\mathrm{CpG}-\mathrm{ODN}$ induced partial protection and enhanced immune response against murine model of leishmaniasis. Exp Parasitol. 2011;129(2):107-114

152. Tafaghodi M, Sajadi Tabasi SA, Payan M. Alginate microsphere as a delivery system and adjuvant for autoclaved Leishmania major and Quillaja saponin: preparation and characterization. Iran J Pharm Sci. 2007;3(2):61-68.

153. Tafaghodi M, Tabassi SAS, Jaafari MR. Induction of systemic and mucosal immune responses by intranasal administration of alginate microspheres encapsulated with tetanus toxoid and CpG-ODN. Int J Pharm. 2006;319(1-2):37-43.

154. Mehravaran A, Jaafari MR, Jalali SA, Khamesipour A, Tafaghodi M, Hojatizade $\mathrm{M}$, et al. The role of surface charge of ISCOMATRIX nanoparticles on the type of immune response generated against Leishmaniasis in BALB/c mice. Nanomed J. 2015;2(4):249-260.

155. Sabur A, Asad M, Ali N. Lipid based delivery and immuno-stimulatory systems: master tools to combat leishmaniasis. Cell Immunol. 2016; 309:55-60.

156. Zambrano-Villa S, Rosales-Borjas D, Carrero JC, Ortiz-Ortiz L. How protozoan parasites evade the immune response. Trends Parasitol. 2002; 18(6):272-278.

157. Reed SG, Coler RN, Mondal D, Kamhawi S, Valenzuela JG. Leishmania vaccine development: exploiting the host-vector-parasite interface. Expert Rev Vaccines. 2016;15(1):81-90.
Drug Design, Development and Therapy

\section{Publish your work in this journal}

Drug Design, Development and Therapy is an international, peerreviewed open-access journal that spans the spectrum of drug design and development through to clinical applications. Clinical outcomes, patient safety, and programs for the development and effective, safe, and sustained use of medicines are the features of the journal, which
Dovepress

has also been accepted for indexing on PubMed Central. The manuscript management system is completely online and includes a very quick and fair peer-review system, which is all easy to use. Visit http://www.dovepress.com/testimonials.php to read real quotes from published authors. 